\title{
La legislazione italiana sulla fecondazione assistita: tra norme restrittive e interpretazioni giuridiche
}

\author{
Giuseppe Benagiano ', Sabina Carrara ', Valentina Filippi ${ }^{1}$ \\ 1 Dipartimento di Ginecologia e Ostetricia, Università Sapienza, Roma
}

\begin{abstract}
The Italian Law 40/2004 regulates assisted reproduction technology; it states that no more than three embryos must be created at any one time; that all the embryos created must be transferred together, and that embryo cryopreservation is forbidden. Six years after its promulgation, the debate over this issue is still heated. On 8 May 2009, the Italian Constitutional Court declared that some parts of the law are unconstitutional. The article highlights the key points of the law and their implications, underlining the importance of coming to an agreement between the parties to the case, with the final aim of protecting both women's and children's health.
\end{abstract}

Keywords: Italian Law 40/2004, assisted reproduction technology

The Italian Law on assisted reproduction technology: between strict regulations and juridical constructions Pratica Medica \& Aspetti Legali 2010; 4(2): 73-77

\section{INTRODUZIONE}

È a tutti noto che la legge 40/2004 [1] che regola le attività connesse alla cosiddetta procreazione medico-assistita (internazionalmente nota con l'acronimo ART) e in particolare alle varie metodiche di fertilizzazione in vitro, seguita da trasferimento dell'embrione nell'utero materno (FIVET $\mathrm{o}$, in inglese, IVF-ET), contiene norme considerate restrittive dalla maggioranza degli "addetti ai lavori”. A questa obiezione, i fautori della legge rispondono con due ordini di argomenti: uno di carattere bioetico, la necessità cioè di salvaguardare la vita di ogni embrione una volta concepito; l'altro di carattere giuridico e cioè il fatto che la legge ha con successo superato lo scoglio del referendum abrogativo e ha quindi ottenuto anche la "legittimità popolare".

Recentemente, i termini del problema sono però mutati per via di una decisione della Corte Costituzionale che, con sentenza dell'8 maggio 2009 [2], ha abrogato parti significative e controverse della legge, dando forza agli argomenti di natu- ra medico-scientifica sviluppati contro alcune sue parti dagli addetti ai lavori [3]. La Corte ha focalizzato la sua attenzione principalmente su due punti che cercheremo di illustrare utilizzando ragioni di natura soprattutto medico-biologica, perché è su di esse che si è focalizzata l'attenzione della Corte.

\section{" L'IMPERATIVO DELLA "BUONA PRATICA MEDICA"}

Il primo punto preso in considerazione dalla Corte nella sua sentenza riguarda la necessità che la legge affidi allo specialista che pratica la FIVET un ruolo centrale, non sostituibile con dettati legali. Questo perché compito del medico è da sempre quello di esercitare "l'arte medica" secondo criteri di appropriatezza e correttezza. Oggi questo si ottiene, da un lato seguendo linee guida nazionali 
e internazionali che in molti campi sono disponibili e indicano chiaramente quale deve essere la condotta da seguire; dall'altro, adattando opportunamente al singolo caso ogni procedura, perché è responsabilità primaria del medico trattare una persona, non una malattia.

Il grande problema posto dalla legge 40/2004 risiede proprio nel fatto che - come abbiamo scritto ancor prima che la legge fosse promulgata - essa rischia in alcuni casi di imporre la cosiddetta malpractice, cioè una condotta medica non solo non ottimale, ma addirittura sanzionabile legalmente. Nel 2002 uno degli Autori aveva infatti dichiarato il proprio «turbamento di fronte ad un testo che dopo attenta lettura - appare imporre per legge l'obbligo di utilizzare una metodica (quella della riproduzione medico-assistita) con modalità non solo non ottimali, ma addirittura tali da creare validamente l'accusa di essere contrarie all'etica ed alla deontologia professionale, perché sottopongono le donne che desiderano avvalersene a rischi aumentati e a risultati ridotti» [4].

Questa realtà è stata riconosciuta dalla Corte Costituzionale che ha dichiarato incostituzionale l'articolo 14, comma 2 per quanto concerne l'espressione «un unico e contemporaneo impianto, comunque non superiore a tre» e il comma 3 , in quanto si è omesso di affermare che il trasferimento debba avvenire senza pregiudizio per la salute della donna.

Riteniamo che il principio fondamentale affermato nella sentenza della Corte sia proprio quello della supremazia del giudizio del medico curante, naturalmente quando esso sia conforme alla migliore pratica del momento.

Non può quindi - a giudizio della Corte - essere permesso esporre una donna a procedure non necessarie (la ripetizione della procedura di stimolazione), come potrebbe accadere di fronte a un divieto assoluto di congelamento degli embrioni. Né è lecito far correre a chi si sottopone a una metodica FIVET dei rischi che, prendendo le opportune precauzioni, sono evitabili, come può avvenire a causa dell'obbligo previsto dalla legge 40/2004 di produrre non più di tre embrioni e di trasferire in ogni caso tutti gli embrioni prodotti. Questa norma obbliga il medico a ignorare le probabilità di concepimento di ogni singola donna (probabilità che è legata in primis alla sua età), con il conseguente rischio, nelle più giovani, di gravidanze multiple. Sul versante opposto, lo stesso dettame può diminuire le probabilità di avere un figlio per le donne in età superiore ai 40 anni che si gioverebbero della possibilità di fecondare più di tre embrioni [5].

La Corte ha anche sottolineato un fatto che molti non conoscono: per anni la giurisprudenza costituzionale italiana ha costantemente sottolineato come il progresso scientifico e sperimentale in campo medico ponga dei limiti "di fatto" al potere discrezionale del legislatore, come dimostrato ad esempio da due sentenze del 2002 e del 2003 [6,7]. È questo consolidato principio costituzionale che indica come punto di riferimento invalicabile anche per il legislatore l'autonoma responsabilità del medico curante di scegliere il trattamento ritenuto migliore per ogni singolo caso. Naturalmente ciò deve avvenire previo accertamento dell'indispensabile "consenso informato" della donna. Da sottolineare che questa infelice espressione (frutto, come la tragicomica espressione "porta allarmata" che leggiamo negli aeroporti, di una cattiva traduzione dall'inglese) non significa che il medico ha semplicemente l'obbligo di informare la paziente. Al contrario, egli ha l'obbligo di assicurarsi che la donna abbia compreso quanto le è stato illustrato. È per questo che si dovrebbe usare per definirlo l'espressione "consenso cosciente".

È giusto riconoscere che la logica impeccabile e quindi la forza del ragionamento seguito dalla Corte sono state riconosciute anche da chi ha sempre difeso la legge 40/2004 nella sua originaria stesura. Infatti, sotto il titolo "Consulta: sugli embrioni la parola spetta al medico", Negrotti ha scritto sull'Avvenire: "È necessario stabilire un equilibrio tra il diritto alla salute della donna e quello alla vita del bambino» [8]. Tanto più che la Corte non ha affatto modificato il principio basilare contenuto nella legge e cioè che non si possa procedere al trasferimento di più di tre embrioni.

\section{LA DOVEROSA PROTEZIONE DEI DIRITTI DEL CONCEPITO}

I difensori della legge 40/2004 si sono opposti alle modifiche approvate dalla Corte Costituzionale e lo hanno fatto nel nome della difesa della vita e dei diritti del concepito. L'argomento principe da loro utilizzato è stato chiaramente espresso dall'eurodeputato Carlo Casini, Presidente del Movimento per la Vita italiano; Casini ha lamentato che il I Rapporto Ministeriale sull'applicazione della legge 40/2004 ha omesso qualsiasi riferimento agli effetti che la nuova legislazione ha avuto sulla "difesa della vita”. Egli ha scritto che, in conseguenza del dettato della nuova legge, nel 2005, 106.833 ovociti sono stati eliminati a causa della proibizione di creare più di tre embrioni, argomentando che, senza la legge, tutti questi ovociti (o perlomeno una gran parte di essi) sarebbero stati fecondati e "immediatamente distrutti". In altre parole, la legge ha evitato che un centinaio di migliaia di "esseri umani" fossero generati e immediatamente lasciati morire [9].

Questa affermazione tuttavia ignora un dato biologico oggi ben documentato: è "madre natura" che 
crea miliardi di vite umane destinate - secondo la definizione di Casini - a una distruzione pressoché immediata. È stato infatti dimostrato attraverso dati epidemiologici, embriologici, biochimici e clinici che, nella specie umana, la maggioranza degli ovociti fecondati non giunge al termine di gravidanza e non produce quindi un bambino. In massima parte questa perdita avviene nel periodo embrionale cosiddetto pre-clinico (e cioè prima che la donna sappia di essere incinta) [10].

Molto opportunamente la Corte Costituzionale ha preso in seria considerazione questo fenomeno biologico, traendone la conclusione che la protezione dell'embrione, seppur fatto positivo e da sostenere, non può essere assoluta. $E$ lo ha fatto utilizzando proprio il testo della legge 40/2004, testo che limitando a tre (e non ad uno!) il numero degli embrioni da produrre, riconosce implicitamente che nella maggioranza dei casi due dei tre embrioni andranno spontaneamente perduti. È quindi proprio la legge 40/2004 che dichiara l'impossibilità biologica di una "protezione assoluta" di ogni embrione, indipendentemente dal fatto che esso sia stato prodotto per via naturale o attraverso una tecnica FIVET.

L'argomento è certamente altamente controverso e su di esso sono stati scritti fiumi di parole. Riassumendo, con Pennings e de Wert [11], le varie posizioni etiche si può dire che a un estremo vi sono coloro che considerano un embrione una "non-persona" e quindi un'entità priva di status morale indipendente; secondo costoro, il rispetto e la cura con cui gli embrioni vanno trattati dipende esclusivamente dai desiderata delle persone che hanno fornito i gameti [12]. All'altro estremo vi è invece chi considera ogni embrione e persino l'uovo fecondato una persona, o perlomeno una "persona potenziale" per via del suo potenziale intrinseco a divenire una persona [13]. La Corte Costituzionale italiana, come la maggioranza dei legislatori europei, si è posta in una posizione intermedia che, distinguendo implicitamente lo stadio di sviluppo raggiunto da un embrione, definisce gli embrioni ottenibili con la FIVET (cioè quelli allo stadio pre-impianto) come aventi diritto a forme di protezione che tuttavia, essendo diverse da quelle di una persona, non sono assolute.

Argomentando contro l'idea che gli embrioni siano moralmente equivalenti a dei bambini o a degli adulti, come sostengono molti appartenenti al cosiddetto Movimento "Pro-life", Greene ha recentemente sottolineato come, sulla base delle stime correnti per la crescita della popolazione mondiale, si dovrebbe concludere che ogni anno vi è una «perdita catastrofica di qualcosa come 100 milioni di vite "umane" nel mondo" [14]. Come mai allora, argomenta Greene, nell'establishment medico-scientifico mondiale, nessuno ha mai proposto di concentrare fondi e progetti di ricerca su questo fenomeno? Egli continua sostenendo che «questa indifferenza mo- rale non si giustifica sostenendo che le morti embrionarie sono il risultato di un processo "naturale", ad esempio di quello dell'alterazione cromosomica detta aneuploidia». Infatti, moltissime patologie considerate minacce globali per la salute sono il risultato di processi naturali. Né sembra valido l'argomento che le morti dovute a malattie o ad alterazioni cromosomiche non sono intenzionali.

Una posizione diametralmente opposta è quella sostenuta invece dalla dottrina cattolica la quale insegna che il frutto della procreazione umana richiede, dal primo istante della sua esistenza, lo stesso rispetto senza pre-condizioni dovuto a ogni essere umano nella sua totalità fisica e spirituale. Quest'affermazione deriva dallo speciale coinvolgimento creativo di Dio che rende ogni forma di vita umana sacra e inviolabile. Questa dottrina accetta il fatto che non sia possibile determinare il momento preciso in cui l'anima viene infusa in un embrione ma, anche supponendo un'animazione ritardata, afferma che fin dall'inizio vi è una nuova vita che si prepara a ricevere un'anima immortale che completi la natura umana ricevuta dai propri genitori [13]. In quest'ottica, il fatto che una buona parte degli embrioni non continuerà nel suo sviluppo, è irrilevante: poiché noi non conosciamo quali tra loro abbiano o meno capacità di svilupparsi, dobbiamo proteggerli tutti [15].

\section{- L'APPROVAZIONE POPOLARE DELLA LEGGE 40}

Tra gli argomenti con i quali i difensori della legge 40/2004 hanno reagito alla sentenza della Corte Costituzionale del 2009, ve ne è uno che merita particolare attenzione, perché implica l'accusa che la Corte si sia resa colpevole di aver stravolto la volontà popolare.

Non essendo di formazione giuridica, non possiamo discutere il valore, i limiti o i mezzi attraverso i quali la volontà popolare può legittimamente esprimersi in Italia. Ci limitiamo quindi ad alcune osservazioni di carattere generale, riprendendo quanto abbiamo già scritto [16]. Crediamo si debba partire dal fatto che astenersi dal voto in un referendum è un'opzione assolutamente legittima, anche se - in pratica - utilizzando questo strumento, chi è contrario ha dalla sua l'astensione fisiologica che nell'Italia moderna si posiziona tra il 25 e il $30 \%$. Questo significa che basta un 20-25\% di astensioni "mirate" per invalidare qualunque referendum.

Tuttavia, nel caso del referendum sulla legge $40 / 2004$, questo argomento non può essere utilizzato, visto che l'astensione ha sfiorato il $75 \%$ ! Nel caso specifico, semmai, andrebbe stigmatizzato il fatto che astenersi su un argomento di così im- 
portante valore etico equivale a rinunciare a una battaglia di principio, ricorrendo invece a un espediente politico.

Noi preferiamo partire da una constatazione: il contenuto dei referendum era tale che definirlo "astruso" è riduttivo. Gli italiani non hanno capito, né potevano capire, leggendo i quesiti proposti, di cosa si stava parlando e su cosa dovevano decidere. In questa situazione l'astensione era, occorre ammetterlo, un'opzione valida. E di questo i promotori devono farsi carico.

Inoltre il nostro Paese è forse il più critico in Europa nei confronti del vertiginoso progresso della scienza e si è venuto sviluppando un movimento di rigetto che va ben al di là della riproduzione medico-assistita. Anche se gli argomenti contro molti dei recenti sviluppi della genetica, dagli organismi geneticamente modificati, alla ricerca sugli embrioni, alla clonazione, vengono in generale dismessi dal mondo della ricerca come "non scientifici", i ricercatori sembrano ignorare il fatto che, anche se non validi sul piano scientifico, questi argomenti inevitabilmente finiscono per influenzare le decisioni politiche molto di più delle osservazioni puramente tecniche [17].

Occorre quindi che il mondo della ricerca "scenda dalla cattedra", riconosca di avere il dovere di spiegare a tutti ciò che fa, ascolti le critiche e si prenda carico anche delle paure e di rigetti considerati irrazionali. Solo così un Paese può raggiungere il consenso necessario a fare quei passi avanti che - in teoria - la scienza oggi gli permetterebbe di fare.

\section{CONCLUSIONI}

Sei anni dopo l'approvazione della legge 40/2004 e dopo decenni di discussioni, in Italia il dibattito sulla procreazione medico-assistita è ancor lungi dall'essersi concluso. Continuano infatti i ricorsi ai Tribunali da parte coppie che chiedono autorizzazioni in deroga alla legge e continuano le sentenze che si discostano da quella che chiameremmo "l'interpretazione letterale" della legge.

Questa situazione è tutt'altro che soddisfacente e, per parte nostra, possiamo solo auspicare - come da anni non si stanca di fare il Presidente della Repubblica, Giorgio Napolitano - che i toni si smorzino, che invece di dividersi su tutto, gli opposti schieramenti cerchino un terreno di intesa, un minimo comune denominatore che, come ha fatto la Corte Costituzionale, abbia come valori supremi la salute della donna che si sottopone alla FIVET (e, più in generale, a tutte le pratiche di ART) e quella del nuovo essere che sarà concepito.

\section{DISCLOSURE}

Gli Autori dichiarano di non avere conflitti di interesse di natura finanziaria.

\section{BIBLIOGRAFIA}

1. Repubblica Italiana, legge 19 febbraio 2004, "Norme in materia di procreazione assistita". Gazzetta Ufficiale della Repubblica Italiana. Serie generale 45: 5-12, 24.2.2004

2. Corte Costituzionale della Repubblica Italiana, sentenza 151/2009, nei giudizi di legittimità costituzionale dell'articolo 6, comma 3, e dell'articolo 14, commi 1, 2, 3 e 4, della legge 19 febbraio 2004, n. 40 ("Norme in materia di procreazione medicalmente assistita")

3. Benagiano G, Gianaroli L. The Italian Constitutional Court modifies Italian legislation on assisted reproduction technology. Reproductive BioMedicine Online 2010; 20: 398-402

4. Benagiano G. Una legge che impone la malpractice? Osservazioni di un ginecologo sulla proposta di legge. Bioetica 2002; 10: 561-3

5. Benagiano G, Gianaroli L. The new Italian IVF legislation. Reproductive Biomedicine Online 2004; 9: 117-25

6. Corte Costituzionale della Repubblica Italiana, sentenza 282/2002 nel giudizio di legittimità costituzionale della legge della Regione Marche 13 novembre 2001 n. 26, recante "Sospensione della terapia elettroconvulsivante, della lobotomia prefrontale e transorbitale e altri simili interventi di psicochirurgia”. Disponibile su: http:/www.giurcost. org/decisioni/2002/0282s-02. html (ultimo accesso: aprile 2010)

7. Corte Costituzionale della Repoubllica Italiana, sentenza 338/2003 nel giudizio di legittimità costituzionale degli articoli 4, 5 e 6 della legge della Regione Piemonte 3 giugno 2002, n. 14, recante "Regolamentazione sull'applicazione della terapia elettroconvulsivante, la lobotomia prefrontale e transorbitale ed altri simili interventi di psicochirurgia”. Disponibile su: http://www. giurcost.org/decisioni/2003/0338s-03.html (ultimo accesso: aprile 2010)

8. Negrotti E. Consulta: sugli embrioni la parola spetta al medico. Avvenire 9 maggio 2009

9. Casini C. A tre anni dalla Legge 40/2004 sulla "procreazione medicalmente assistita": un esame critico della relazione del Ministro della Salute. Medicina e Morale 2007; 57: 691-715 
10. Benagiano G, Farris M, Grudzinskas G. The fate of fertilised human oocytes. Reproductive Biomedicine Online 2010 [In corso di stampa]

11. Pennings G, de Wert G. Evolving ethics in medically assisted reproduction. Human Reproduction Update 2003; 9: 397-404

12. McCullough LB, Chervenak FA. Ethics in obstetrics and gynecology. New York and Oxford: Oxford University Press, 1994

13. Congregazione per la Dottrina della Fede. Istruzione: Donum vitae. Il rispetto della vita umana nascente e la dignità della procreazione. 1987. Disponibile su: http://www.vatican.va/roman_curia/congregations/cfaith/documents/rc_co n_cfaith_ doc_19870222_respect-for\%20human-life_it.html (ultimo accesso: aprile 2010)

14. Green RM. Embryo as epiphenomenon: some cultural social and economic forces driving the stem cell debate. Journal of Medical Ethics 2008; 34: 840-4

15. Benagiano G, Mordini E, Pera A. Il dibattito nella Bioetica: sulla natura del cosiddetto pre-embrione. Medicina Morale 1993; 43: 783-7

16. Benagiano G. The four referendums attempting to modify the restrictive Italian IVF legislation failed to reach the required quorum. Reproductive Biomedicine Online 2005; 11: 279-81

17. Benagiano G, Farris M. Public health policy and infertility. Reproductive Biomedicine Online 2003; 7: 606-14

\section{CORRESPONDING AUTHOR}

Prof. Giuseppe Benagiano.E-mail: giuseppe.benagiano@uniroma1.it 